\title{
CYL-02 Plasmid DNA
}

National Cancer Institute

\section{Source}

National Cancer Institute. CYL-02 Plasmid DNA. NCI Thesaurus. Code C95716.

A gene transfer preparation of a plasmid DNA encoding mouse somatostatin receptor subtype 2 (sst2) and a fusion protein of human deoxycytidine kinase (DCK) and uridine monophosphate kinase (UMK), complexed to a synthetic polycationic carrier, polyethylenimine, with antineoplastic adjuvant application. Upon administration, CYL-02 plasmid DNA expresses DCK::UMK fusion protein that converts gemcitabine into its toxic phosphorylated metabolite. Expression of sst2 protein by this agent could induce both antioncogenic and local antitumor bystander effects. A loss of sst2 gene expression often is found in pancreatic and colorectal cancers, and is the receptor for somatostatin which negatively regulates a number of processes such as epithelial cell proliferation. Combination effects of these gene products allows for less chemotherapy to cause tumor cell lysis in not only the original tumor, but in distant tumors as well. 